\title{
Isolated Oropharyngeal Dysphagia as the Initial Presentation of Anti-SRP Immune-Mediated Necrotizing Myopathy
}

Pat Korathanakhun, M.D., Thanyalak Amornpojnimman, M.D.

Unit of Neurology, Division of Internal Medicine, Faculty of Medicine, Prince of Songkla University, Hat Yai, Songkhla 90110, Thailand.

Received 24 February 2020 • Revised 23 May 2020 • Accepted 3 June 2020 • Published online 25 August 2020

\begin{abstract}
:
A 51-year-old male initially presented with a progressive course of isolated oropharyngeal dysphagia prior to the clinical course of painful symmetrical proximal muscle weakness without sensory deficit which rendered him to wheelchairbound status within 5 months. The physical examination revealed symmetrical proximal muscle weakness without sensory symptoms. The initial serum creatine kinase was extremely high and the electrodiagnostic study revealed a myopathic pattern. A muscle biopsy of the left biceps suggested a diagnosis of immune-mediated necrotizing myopathy (IMNM) and the serum anti-signal recognition particle (SRP) autoantibody was finally detected. This case presented a rare form of anti-SRP IMNM in which isolated oropharyngeal dysphagia preceded the onset of proximal muscle weakness.
\end{abstract}

Keywords: anti-SRP autoantibody, immune-mediated necrotizing myopathy, oropharyngeal dysphagia

Contact: Thanyalak Amornpojnimman, M.D.

Unit of Neurology, Division of Internal Medicine, Faculty of Medicine,

Prince of Songkla University, Hat Yai, Songkhla 90110, Thailand.

E-mail: athanyal@medicine.psu.ac.th

This is an open access article under the CC BY-NC-ND license

(http://www.jhsmr.org/index.php/jhsmr/about/editorialPolicies\#openAccessPolicy). 


\section{Introduction}

With advanced techniques of muscle pathology and serologic studies, immune-mediated necrotizing myopathy (IMNM) is an emerging myositis in this era. The clinical course of chronic, progressive, painful, symmetrical proximal muscle weakness, with elevated serum creatine kinase; without skin signs, are commonly recognized by neurologists and rheumatologists and raises the suspicion for immune-mediated myopathy. Although, oropharyngeal muscles can be involved in IMNM, oropharyngeal dysphagia typically presents in the late stage of the disease. Isolated oropharyngeal dysphagia as a presenting symptom, which may mislead clinicians to other groups of neuromuscular diseases; therefore, causing delayed diagnosis and increased morbidity. In this case report, the authors present a case of anti-signal recognition particle IMNM in a patient, who initially presented with isolated, progressive oropharyngeal dysphagia prior to the involvement of proximal muscle.

\section{Case report}

A 51-year-old Thai male initially presented with difficulty of swallowing solid food for a couple of weeks which progressive worsened to complete oropharyngeal dysphagia that led to the requirement of nasogastric tube insertion without dyspnea within 1 month. During this period, the patient reported normal motor function in all extremities. Later on, he developed slowly progressive symmetrical proximal muscle weakness with muscle sore. At the first medical visit to the primary doctor, the serum creatine kinase (CK) level was 8,336 international units per liter (IU/) and all potential drugs induced myopathy including colchicine and simvastatin were immediately withheld. However, the clinical course of motor weakness worsened to wheelchair-bound status within 5 months. Moreover, he also experienced both anorexia and significant weight loss. The review of system including rheumatologic, pulmonary, cardiac, endocrine, and renal symptoms were within normal limits. None of his family members had any history of neuromuscular disease.

The physical examination revealed generalized muscle atrophy, the proximal muscle and neck muscle weakness with the Medical Research Council (MRC) score of grade II while the distal muscle power of the MRC score remained as grade $\mathrm{V}$ in all extremities. Deep tendon reflexes also decreased with neither deficit of pinprick sensation nor proprioception. The other neurological signs and general systemic signs were within normal limits. Neither heliotrope nor grotton's papule were detected. Regarding the laboratory results, the serum CK level was 12,848 IU $\Lambda$. The motor nerve conduction study revealed decreased compound-muscle action potential amplitude with preserved distal latency, nerve conduction velocity of the left medial, left ulnar, and both tibial nerves. The sensory nerve conduction study was within normal limits. The needle electromyography elicited small-amplitude, short-duration motor unit and fibrillation potentials with positive sharp waves at the right vastus medialis, deltoid, and medial gastrocnemius muscles. Thyroid function test and antinuclear antibody were normal.

Therefore, biopsy of the left biceps muscle was done and the pathological finding revealed scattered areas of muscle necrosis with few lymphocytic infiltration (Figures 1A-B). Neither perimysial nor perifascicular atrophy were found. The immunohistochemical staining showed abundant Cluster of Differentiation 68+ macrophages surrounding the muscle necrotic area with paucity of Cluster of Differentiation 4+, Cluster of Differentiation 8+, and Cluster of Differentiation 3+ lymphocytes (Figures 1C-F). These findings were consistent with IMNM. In addition, the antisignal recognition particle (SRP) autoantibody was positive in the serum test of the myositis antibodies panel. The diagnosis of anti-SRP IMNM was made. Regarding associated occult malignancy, further investigations including 
computed tomography of the chest and abdomen, earnose- throat examination, and esophagogastroduodeno- scopy with colonoscopy, were performed with all negative results.

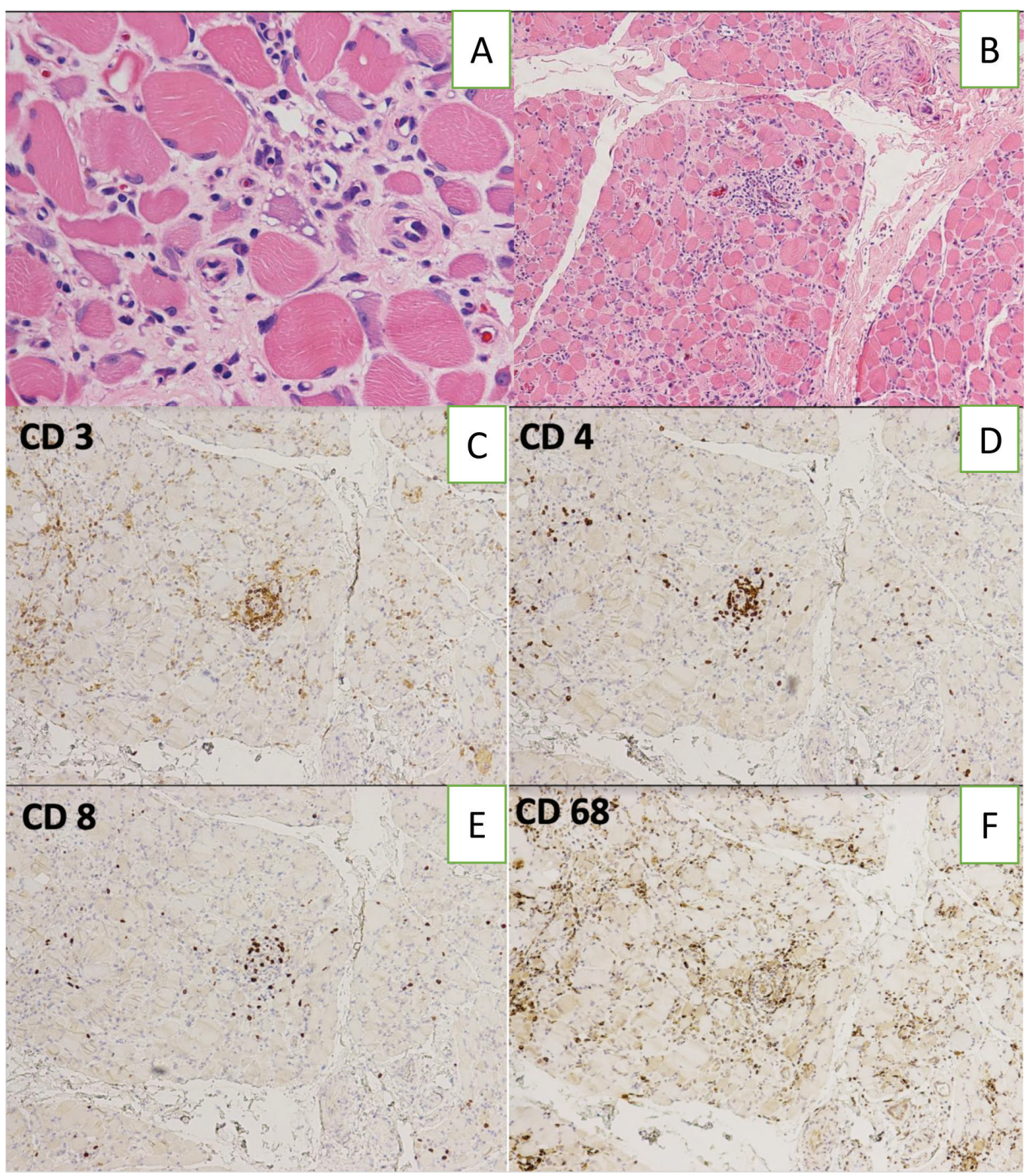

Figure 1 Histopathological finding of the left biceps muscle biopsy (A-B). The hematoxylin and eosin stain staining in low and high power field showed muscle atrophy, compensatory hypertrophy, and inflammatory cells in the endomysium with some areas of necrotic muscle fibers; (C-F) Immunohistochemical staining showed CD68+ macrophages diffusely surrounding the areas of muscle necrosis with a paucity of CD4+, CD8+, and CD3+ lymphocytes. 
The treatment was initiated with 5 consecutive day of intravenous methylprednisolone 1,000 milligram followed by 1 milligram/kilogram/day of prednisolone. In addition to the corticosteroid, monthly intravenous cyclophosphamide 600 milligram/square meter of body surface area was also prescribed. After aggressive treatment for two months, the disabling oropharyngeal dysphagia dramatically resolved along with an improvement of motor power of all extremities. The serum CK also decreased to a level of 440 IU $/$.

\section{Discussion}

Idiopathic inflammatory myopathies are rare autoimmune disorders affecting the skeletal muscle tissues which are technically recognized as 5 distinctive diseases: polymyositis, dermatomyositis, inclusion body myositis, overlap syndrome with myositis including anti-synthetase syndrome and IMNM. Although IMNM accounted for only one-fifth of all inflammatory myopathies, the clinicians should not miss the diagnosis due to the aggressive natural history and also the refractoriness to treatment.

The well-recognized clinical presentations of IMNM consist of painful symmetrical proximal muscle weakness with elevated serum CK. The history of rapid progressive deterioration after statin exposure without improvement after withholding statin therapy can raise the suspicion of IMNM associated with anti-3-hydroxy-3-methylglutarylCoA reductase (HMGCR). In terms of anti SRP myopathy, the clinical features of severe weakness, neck weakness, dysphagia, and muscle atrophy were observed more frequently than in patients with anti-HMGCR antibodies. ${ }^{1,2}$

SRP autoantibody may present in some cases of polymyositis and dermatomyositis. Patients with a positive anti-SRP test usually had more rapid onset and severe disease than other autoimmune myopathy cases. Furthermore, patients with other autoimmune myopathy often had interstitial lung, arthritis and skin involvement. Therefore, the interpretation of serology results in inflammatory myo- pathies should be consided with clinical presentations and pathological findings. ${ }^{1,3}$

The prevalence of oropharyngeal dysphagia ranged widely among IMNM patients from about 10.0 to $70.0 \%$ along the clinical course. ${ }^{1,2}$ Oropharyngeal dysphagia developed as a result of immune-mediated inflammation that occurred in the skeletal muscle of the oropharyngeal, laryngeal, and esophageal muscles causing incoordination of swallowing. Apart from the dysphagia, laryngeal muscle involvement can cause hoarseness, nasal voice, and respiratory dysfunction (aspiration, respiratory muscle deficiency). ${ }^{4,5}$ Regarding dysphagia as initial presenting symptom in inflammatory myositis, $42.0 \%$ of whom were diagnosed as having inclusion body myositis, $22.0 \%$ in polymyositis and overlap syndrome. ${ }^{6}$ Jaradeh also reported that dysphagia can be an initial symptom in inclusion with body myositis; especially in elderly patients. ${ }^{4} \mathrm{~A}$ cohort study conducted in the muscle biopsy- oriented registry in Japan revealed that dysphagia was detected in $68.0 \%$ of the anti-SRP IMNM group compared with $44.0 \%$ of the anti-HMGCR group. ${ }^{1}$ However, most of the IMNM patients experienced oropharyngeal dysphagia as a late symptom in the advanced stage of disease. Isolated oropharyngeal dysphagia rarely presented as the initial symptom in merely $7.0 \%$ of IMNM patients. ${ }^{2}$

Based on the current evidence, no randomized trial has demonstrated the optional immunosuppressive treatment regimen for IMNM. However, the IMNM working group recommended methotrexate, rituximab and intravenous immunoglobulin (IVIg) as an induction and a maintenance treatment in IMNM. $^{7}$ In health-economic context of Thailand, prescribing IVIg or rituximab as first line therapy remain problematic due to the reimbursement limitation.

Occult malignancy was found in approximately $8.0 \%$ of anti-SRP IMNM myopathy patients at the time of IMNM diagnosis. However, regular tumor surveillance 
should be conducted despite the negative results of an initial extensive evaluation as the mean duration between the diagnosis of cancer and the myopathy was 6-7 years and half of the malignancies occurred within 3 years of or before the diagnosis of anti-SRP IMNM positive patients. ${ }^{8}$ Some reports found that dysphagia was seen in patients with internal malignancy, anti-transcription intermediary factor 1 gamma antibody in dermatomyositis patients, but this association was not found in anti-SRP IMNM positive patients. $^{9}$

\section{Conclusion}

Though it's rare, isolated oropharyngeal dysphagia can present as the initial symptom in IMNM which is in contrast to the conventional symptoms in the late stage of the disease. Early recognition of this atypical presentation can lead to a prompt diagnosis and aggressive treatment to maximize the clinical outcome.

\section{Acknowledgement}

The authors would like to cordially thank Prof. Winyou Mitranun for his courtesy in reviewing the pathological slides. The english editing of this manuscript by Mr. Glenn Shingledecker was also appreciated.

\section{Funding sources}

This manuscript was funded by the Faculty of Medicine, Prince of Songkla University, Thailand.

\section{Conflict of interest}

The authors declare no conflict of interest in this manuscript.

\section{References}

1. Watanabe $\mathrm{Y}$, Uruha A, Suzuki S, Nakahara J, Hamanaka K, Takayama $\mathrm{K}$, et al. Clinical features and prognosis in antiSRP and anti-HMGCR necrotizing myopathy. J Neuro Neurosurg Psychiatry 2016;87:1038-44.

2. Suzuki S, Nishikawa A, Kuwana M, Nishimura H, Watanabe Y, Nakahara J, et al. Inflammatory myopathy with anti-signal recognition particle antibodies: case series of 100 patients. Orphanet J Rare Dis 2015;10:61.

3. Casciola-Rosen L, Mammen AL. Myositis autoantibodies. Curr Opin Rheumatol 2012;24:602-8.

4. Jaradeh S. Muscle disorders affecting oral and pharyngeal swallowing. GI motility online [homepage on the Internet]. 2006 [cited 2020 May 18]. Available from: https://www.nature. com/gimo/contents/pt1/full/gimo35.html

5. Ebert EC. Review article: the gastrointestinal complications of myositis. Alimen Phamacol Ther 2010;31:359-65.

6. Oh TH, Brumfield KA, Hoskin TL, Stolp KA, Murray JA, Bassford JR. Dysphagia in inflammatory myopathy: clinical characteristics, treatment strategies, and outcome in 62 patients. Mayo Clin Proc 2007;82:441-7.

7. Allenbach $Y$, Mammen AL, Benveniste O, Stenzel W. $224^{\text {th }}$ ENMC International Workshop: clinico-sero-pathological classification of immune-mediated necrotizing myopathies Zandvoort, The Netherlands, 14-16 October 2016. Neuromuscul Disord 2018;28:87-99.

8. Allenbach $\mathrm{Y}$, Karaen J, Bouvier A, Jooste V, Champtiaux N, Hervier B, et al. High risk of cancer in autoimmune necrotizing myopathies: usefulness of myositis specific antibody. Brain 2016;139:2131-5.

9. Mugii N, Hasegawa M, Matsuchita T, Hamaguchi Y, Oohata S, Okita $\mathrm{H}$, et al. Oropharyngeal dysphagia in dermatomyositis: associations with clinical and laboratory features including autoantibodies. Plos One 2016;10:1371. 